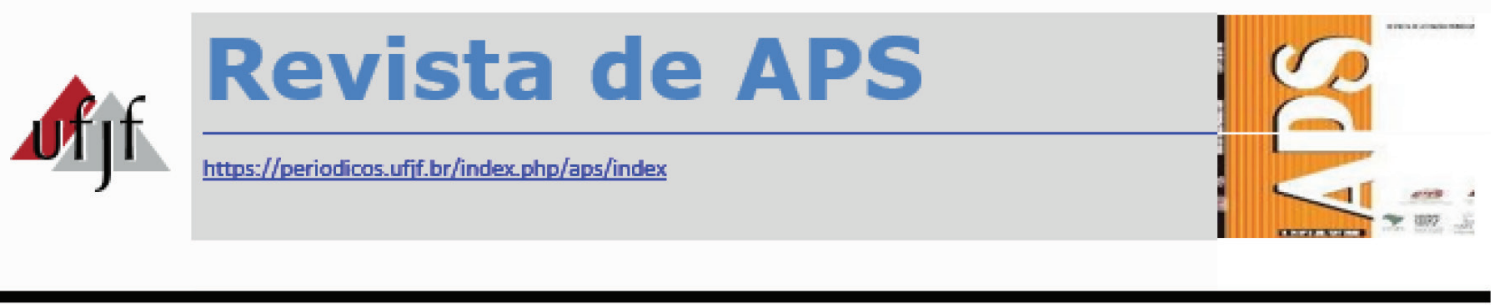

\title{
A educação pelo trabalho na Atenção Primária à Saúde: reflexões sobre a integração ensino-serviço-comunidade
}

\author{
Education through work in Primary Health Care: reflections on teaching- \\ service-community integration
}

\author{
Israel Soares Deagostini ${ }^{1}$, Pedro Henrique Oliveira Lima ${ }^{2}$, Riane Souto Medeiros ${ }^{3}$, \\ Eulilian Dias de Freitas ${ }^{4}$, Lélia Cápua Nunes ${ }^{5}$, Alexandra Paiva Araújo Vieira ${ }^{6}$
}

\begin{abstract}
RESUMO
O presente relato de experiência narra a vivência de inserção de discentes, preceptores e tutores na Atenção Primária à Saúde a partir do Programa de Educação pelo Trabalho para a Saúde - PET-Saúde/GraduaSUS no município de Governador Valadares, Minas Gerais. O grupo tutorial atuou no cotidiano de uma Estratégia de Saúde da Família e realizou diagnóstico e avaliação das fragilidades locais, em conjunto com a equipe de saúde e a comunidade. Em seguida, a partir da construção do planejamento, foi implantado um projeto de intervenção que focou na ampliação do acesso da população às informações sobre a Atenção Primária à Saúde, controle social e Sistema Único de Saúde e na reativação do Conselho Local de Saúde. Ficou evidente a necessidade da continuidade das ações e do fortalecimento da integração ensino-serviço-comunidade, com foco em processos de educação permanente, formação em saúde no cenário de prática, empoderamento dos usuários e melhoria da qualidade e acesso ao SUS.
\end{abstract}

PALAVRAS-CHAVE: Atenção Primária à Saúde. Educação em Saúde. Sistema Único de Saúde (SUS). Estratégia Saúde da Família.

\footnotetext{
${ }^{1}$ Graduando de Medicina/ Universidade Federal de Juiz de Fora - Campus Avançado Governador Valadares (UFJF-GV). E-mail: israeldeagostini@hotmail.com

${ }^{2}$ Graduando de Medicina/ Universidade Federal de Juiz de Fora - Campus Avançado Governador Valadares (UFJF-GV).

${ }^{3}$ Graduada em Educação Física/ Educadora Física do Núcleo de Apoio à Saúde da Família (NASF) de Governador Valadares-MG.

${ }^{4}$ Doutora em Saúde Pública pela Universidade Federal de Minas Gerais. Docente da Universidade Federal de Juiz de Fora - Campus Avançado Governador Valadares.

${ }^{5}$ Doutoranda em Saúde Coletiva na Universidade Estadual Paulista. Docente da Universidade Federal de Juiz de Fora - Campus Avançado Governador Valadares.

${ }^{6}$ Doutora em Parasitologia pela Universidade de Campinas. Docente da Universidade Federal de Juiz de Fora - Campus Avançado Governador Valadares.
} 


\begin{abstract}
This present report brings the students' insertion experience, preceptors and tutors in the Primary Health Care Program of Education for Work and Health PET-Saúde / GraduaSUS in the City of Governador Valadares, Minas Gerais. The group followed the daily life of Family Health Strategy and carried out diagnoses and evaluations of the challenges, working together with the community. Through a carefully constructed plan, an intervention project was implemented. It was focused on expanding the population access to information on Primary Health Care, social control and the Unified Health System and the reactivation of the Local Health Council. It was evident that there is the need of continuity actions and the strengthening of teaching-service-community integration, focusing on processes of permanent education, health education in practices scenarios, empowerment of users and improvement of quality and access to SUS.
\end{abstract}

KEYWORDS: Primary Health Care. Health Education. Unified Health System. Family Health Strategy.

\title{
INTRODUÇÃO
}

A Política Nacional de Educação Permanente em Saúde (PNEPS), criada em 2003, preconiza a formação de estudantes e profissionais no cotidiano do serviço de saúde ${ }^{1}$. Nessa perspectiva, em 2008, a partir do estreitamento do diálogo entre os Ministérios da Saúde e Educação, foi criado o Programa de Educação pelo Trabalho para a Saúde PET-Saúde².

Em 2015 foi lançado o edital do PET-Saúde/GraduaSUS, baseado em três eixos de atuação: mudanças curriculares baseadas nas Diretrizes Curriculares Nacionais (DCN) para os cursos da área da saúde; qualificação da integração ensino-serviço-comunidade; e desenvolvimento da docência e da preceptoria articulada às necessidades do SUS ${ }^{3}$. A Secretaria Municipal de Saúde de Governador Valadares (SMS-GV) e a Universidade Federal de Juiz de Fora - Campus Avançado Governador Valadares (UFJF-GV) foram contempladas com o PET-Saúde/GraduaSUS e organizaram o projeto por meio da formação de grupos tutoriais de trabalho multiprofissionais para atuação no território.

A Atenção Primária à Saúde (APS) foi o cenário do primeiro ano do projeto, uma vez que constitui a porta de entrada do serviço de saúde. A finalidade foi o desenvolvimento de uma atenção integral com resultados na saúde e autonomia dos usuários e nos determinantes e condicionantes de saúde das coletividades, além do estimulo ao pensamento crítico, científico e social dos discentes, baseado na vivência e participação ativa em uma comunidade local $^{4,5}$.

Este relato de experiência possui o objetivo de narrar e refletir sobre a vivência desenvolvida a partir da inserção e atuação de um grupo tutorial do curso de Medicina em uma Unidade de Atenção Primária à Saúde (UAPS) da cidade de Governador Valadares, Minas Gerais. 


\section{DESENVOLVIMENTO}

O PET-Saúde/GraduaSUS foi estruturado a partir da formação de seis grupos tutoriais, que contemplavam os cursos da área da saúde: Educação Física, Farmácia, Fisioterapia, Medicina, Nutrição e Odontologia. A presente experiência foi vivenciada por uma equipe integrante do grupo tutorial do curso de Medicina, composta por três docentes, dois preceptores e dois estudantes do curso de Medicina. Os profissionais eram da área de Fisioterapia, Farmácia e Educação Física. A seleção dos docentes, preceptores e estudantes ocorreu por meio de chamada de interessados nos Departamentos, análise de currículo e processo seletivo, respectivamente. As UAPS foram selecionadas em parceria com a Secretaria de Saúde local, considerando o espaço e demais condições disponíveis para a realização das ações previstas no projeto.

As atividades do grupo tutorial foram divididas em dois momentos: 1) diagnóstico local, observação, identificação de pontos críticos e planejamento junto aos profissionais de saúde e usuários; 2) implantação do plano de intervenção. A intenção foi aperfeiçoar o serviço e fortalecer a integração entre o serviço, o ensino e a comunidade, com eixo na APS.

\section{Diagnóstico local, observação, identificação de pontos críticos e planejamento}

Inicialmente, o grupo procedeu ao reconhecimento do território e realização do diagnóstico situacional. Os estudantes foram inseridos na rotina da equipe de Saúde da Família (eSF) e participaram de momentos de interação com os profissionais e usuários da UAPS para identificação dos problemas locais enfrentados. O Planejamento Estratégico Situacional (PES), importante ferramenta para gestão do SUS, foi utilizado para identificação dos pontos críticos ${ }^{6}$.

O ponto crítico priorizado foi o déficit no conhecimento da população sobre o processo de trabalho da Estratégia Saúde da Família (ESF) e o funcionamento do SUS. A metodologia Espinha de Peixe foi utilizada a fim de elucidar as possíveis causas, consequências e os resultados almejados ${ }^{7}$ (Figura 1). Como causa geral dessa situação foi destacado o reduzido impacto das ações de educação em saúde e como consequência geral a resolutividade limitada dos problemas de saúde da população adscrita, contribuindo para uma visão negativa do SUS.

A imagem-objetivo que se desejava alcançar foi a compreensão da comunidade do processo de trabalho da ESF e do funcionamento do SUS. 
Figura 1 - Espinha de Peixe com foco no ponto crítico priorizado na ESF, Governador Valadares-MG

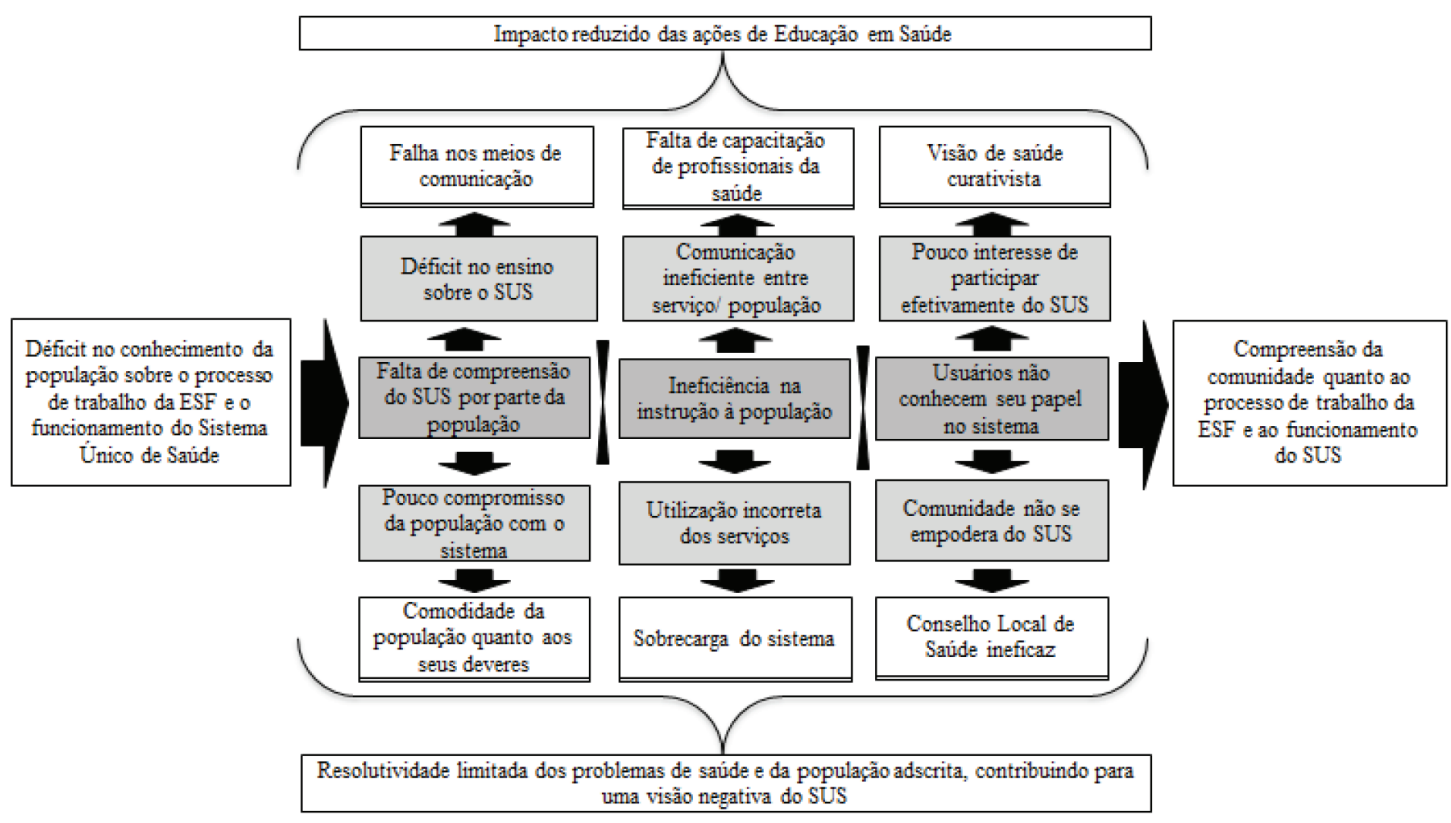

Fonte: elaborada pelos autores

Em seguida foram propostas ações de intervenção para mudança da situação diagnosticada na ESF, com os respectivos objetivos específicos, as operações e ações para alcançá-las, os recursos existentes e ausentes, ator(es) principal(is) e outros parceiros e a análise de viabilidade (Quadro 1). Todas as ações propostas eram viáveis de serem executadas e incorporadas à rotina de trabalho dos profissionais da ESF.

Quadro 1 - Detalhamento das ações de intervenção propostas para a ESF, Governador Valadares-MG

\begin{tabular}{|c|c|c|c|c|}
\hline Ações & Objetivos & $\begin{array}{c}\text { Recursos } \\
\text { necessários }\end{array}$ & $\begin{array}{c}\text { Atores } \\
\text { principais }\end{array}$ & Parceiros \\
\hline $\begin{array}{l}\text {-Realização } \\
\text { de rodas de } \\
\text { conversa } \\
\text {-Confeç̧ão } \\
\text { de material } \\
\text { informativo } \\
\text { (cartazes e } \\
\text { cartilhas) } \\
\text {-Distribuição de }\end{array}$ & $\begin{array}{l}\text {-Melhorar a } \\
\text { compreensão da } \\
\text { organização da APS/ } \\
\text { SUS por parte da } \\
\text { população } \\
\text {-Empoderar o } \\
\text { usuário }\end{array}$ & $\begin{array}{l}\text {-Local para } \\
\text { realização } \\
\text { das rodas de } \\
\text { conversa } \\
\text {-Local para } \\
\text { afixar os } \\
\text { cartazes } \\
\text {-Impressão }\end{array}$ & $\begin{array}{l}\text {-Profissionais } \\
\text { da eSF } \\
\text {-Estudantes do } \\
\text { PET }\end{array}$ & $\begin{array}{l}\text {-Profissionais } \\
\text { do NASF* } \\
\text {-Líderes } \\
\text { religiosos } \\
\text {-Líderes } \\
\text { comunitários }\end{array}$ \\
\hline
\end{tabular}


(Conclusão)

\begin{tabular}{|c|c|c|c|c|}
\hline Ações & Objetivos & $\begin{array}{c}\text { Recursos } \\
\text { necessários }\end{array}$ & $\begin{array}{c}\text { Atores } \\
\text { principais }\end{array}$ & Parceiros \\
\hline $\begin{array}{l}\text {-Realização de } \\
\text { reuniões com os } \\
\text { profissionais da } \\
\text { eSF } \\
\text {-Realização de } \\
\text { orientação nas } \\
\text { consultas e salas } \\
\text { de espera } \\
\text {-Confecção } \\
\text { de material } \\
\text { informativo } \\
\text { (cartazes e } \\
\text { cartilhas) } \\
\text {-Distribuição de } \\
\text { cartilhas }\end{array}$ & $\begin{array}{l}\text {-Levantar o } \\
\text { conhecimento } \\
\text { e alinhar as } \\
\text { orientações junto } \\
\text { aos profissionais da } \\
\text { eSF e estudantes } \\
\text {-Informar a } \\
\text { população sobre o } \\
\text { funcionamento da } \\
\text { APS/SUS } \\
\text {-Ampliar o acesso } \\
\text { à informação sobre } \\
\text { o SUS }\end{array}$ & $\begin{array}{l}\text {-Local para } \\
\text { realização das } \\
\text { orientações } \\
\text {-Local para } \\
\text { afixar os } \\
\text { cartazes } \\
\text {-Impressão }\end{array}$ & $\begin{array}{l}\text {-Profissionais } \\
\text { da eSF } \\
\text {-Estudantes do } \\
\text { PET }\end{array}$ & $\begin{array}{l}\text {-Profissionais } \\
\text { do NASF* } \\
\text {-Líderes } \\
\text { religiosos } \\
\text {-Líderes } \\
\text { comunitários }\end{array}$ \\
\hline $\begin{array}{l}\text {-Realização } \\
\text { de rodas de } \\
\text { conversa } \\
\text {-Confecção } \\
\text { de material } \\
\text { informativo } \\
\text { (cartazes e } \\
\text { cartilhas) } \\
\text {-Distribuição de } \\
\text { cartilhas }\end{array}$ & $\begin{array}{l}\text {-Educar sobre a } \\
\text { participação e } \\
\text { controle social no } \\
\text { SUS } \\
\text {-Fortalecer o } \\
\text { controle social no } \\
\text { SUS }\end{array}$ & $\begin{array}{l}\text {-Local para } \\
\text { realização } \\
\text { das rodas de } \\
\text { conversa } \\
\text {-Local para } \\
\text { afixar os } \\
\text { cartazes } \\
\text {-Impressão }\end{array}$ & $\begin{array}{l}\text {-Profissionais } \\
\text { da eSF } \\
\text {-Estudantes do } \\
\text { PET } \\
\text {-Gestor local }\end{array}$ & $\begin{array}{l}\text {-Profissionais } \\
\text { do NASF* } \\
\text {-Líderes } \\
\text { religiosos } \\
\text {-Líderes } \\
\text { comunitários }\end{array}$ \\
\hline
\end{tabular}

*NASF: Núcleo de Apoio à Saúde da Família

Fonte: elaborada pelos autores

\section{Implantação do plano de intervenção}

Construção de materiais informativos, alinhamento das ações e formação de parcerias

A ação de intervenção foi iniciada por meio da elaboração dos materiais de caráter informativo. Foram confeccionadas e impressas duas cartilhas: a primeira sobre o SUS, com abordagem sobre conceito e funcionamento, conceito de APS e serviços ofertados na ESF; e a segunda sobre o Conselho Local de Saúde (CLS), com foco no conceito, função, papel, importância e composição. De forma complementar foi construída uma apresentação baseada nos temas abordados nas cartilhas para norteamento das rodas de conversas.

Em reuniões com os profissionais da ESF foram alinhadas as estratégias de intervenção e acordadas as ações a serem desenvolvidas, sempre reforçando a importância da parceria entre a Universidade e o cenário de prática, por meio de seus atores. 
Foi aplicado um questionário aos profissionais a fim de nortear a equipe do PET-Saúde/GraduaSUS quanto ao conhecimento a respeito do SUS, para nivelar a informação a ser compartilhada nas orientações à população. Os resultados demonstraram a necessidade da promoção de educação permanente junto aos profissionais. A avaliação do conhecimento dos profissionais acerca do sistema de saúde no qual estão inseridos é importante, uma vez que o entendimento sobre o SUS é essencial na multiplicação de informação a ser compartilhada com os usuários do sistema e na atuação adequada ao modelo proposto na ESF ${ }^{8,9}$. Além disso, a capacitação da equipe acerca das políticas da ESF permite o funcionamento e a consolidação da estratégia e a criação de um vínculo entre usuários e profissionais ${ }^{10}$. A expansão e manutenção dessa prática informativa dos profissionais são indispensáveis para capacitar a população e conscientizá-la, favorecendo sua participação e cooperação na consolidação e desenvolvimento da ESF${ }^{10}$.

Assim, o PET-Saúde/GraduaSUS realizou atividades de educação permanente transversais à inserção dos grupos tutoriais nos cenários de prática, cujas temáticas emergiam das necessidades advindas do cotidiano do serviço, com cerne na vivência da integração ensino-serviço-comunidade.

De forma concomitante, o grupo buscou viabilizar parcerias com lideranças religiosas e comunitárias da área de abrangência da ESF e sondar equipamentos sociais disponíveis, como as igrejas, para a realização de rodas de conversas. Esses locais foram escolhidos em virtude do fácil acesso a um maior contingente de usuários da ESF, uma vez que no entorno não havia escolas ou praças públicas.

Rodas de conversa, divulgação das cartilhas e fortalecimento do Conselho Local de Saúde

As rodas de conversa focaram em temas relacionados ao SUS, à UAPS e ao CLS, inclusive, abordaram informações sobre a realização do processo eleitoral para o novo conselho (Quadro 2). Nesses momentos, as cartilhas elaboradas foram entregues e debatidas. A utilização da cartilha é de grande relevância pedagógica e esse instrumento é uma importante ferramenta na prática de educação ${ }^{11}$. Os materiais também foram distribuídos em outros momentos por meio de integrantes da eSF, NASF e estudantes. 
Quadro 2 - Detalhamento das rodas de conversa realizadas na ESF, Governador Valadares-MG

\begin{tabular}{|l|l|l|l|}
\hline $\begin{array}{l}\text { Roda de } \\
\text { conversa }\end{array}$ & \multicolumn{1}{|c|}{ Objetivo } & \multicolumn{1}{|c|}{$\begin{array}{l}\text { Número de } \\
\text { participantes } \\
\text { (aproximado) }\end{array}$} \\
\hline 1 & $\begin{array}{l}\text { Levantar informações sobre a população } \\
\text { local e o funcionamento do Conselho Local } \\
\text { de Saúde }\end{array}$ & $\begin{array}{l}\text { Trabalhadores da } \\
\text { ESF }\end{array}$ & 12 \\
\hline 3 & $\begin{array}{l}\text { Levantar necessidades da ESF, inclusive para } \\
\text { o pleno funcionamento do Conselho local de } \\
\text { saúde. }\end{array}$ & $\begin{array}{l}\text { Conselheiros de } \\
\text { saúde }\end{array}$ & 3 \\
\hline 4 & $\begin{array}{l}\text { Dialogar sobre o SUS, seu funcionamento } \\
\text { e a importância da participação popular, } \\
\text { principalmente por meio do Conselho local } \\
\text { de saúde. } \\
\text { Abordar o processo eleitoral do novo } \\
\text { conselho. }\end{array}$ & Usuários & 50 \\
\hline $\begin{array}{l}\text { Dialogar sobre o SUS, seu funcionamento } \\
\text { e a importância da participação popular, } \\
\text { principalmente por meio do Conselho local } \\
\text { de saúde. }\end{array}$ & $\begin{array}{l}\text { Usuários } \\
\text { e a importância da participação popular, } \\
\text { principalmente por meio do Conselho local } \\
\text { de saúde. }\end{array}$ & Usuários & 50 \\
\hline
\end{tabular}

Fonte: elaborada pelos autores

Foi elaborado e aplicado um questionário de linguagem simples para levantar o conhecimento dos usuários sobre o SUS, ESF e CLS. Os resultados demonstraram a necessidade de melhorar a comunicação com o usuário para ampliar o conhecimento sobre os serviços ofertados e a rotina da ESF. A transmissão de informações precisa estar ajustada às condições da população e ter o potencial de conscientizar e empoderar os usuários para que tenham participação ativa nos extramuros da unidade de saúde ${ }^{12}$. Apesar das evoluções na participação popular em saúde, há ainda lacunas que necessitam serem preenchidas, pois poucas pessoas têm conhecimento dos mecanismos de atuação na saúde a que possuem direito ${ }^{13}$. Os conselhos de saúde, principalmente nos seus aspectos municipais, necessitam adotar uma abordagem mais focada na comunicação social por meio de metodologias que identifiquem as necessidades e direcionem as medidas a serem adotadas para maior participação popular ${ }^{14}$.

Além dessas ações, foi realizado um contato com os gestores competentes, a fim de compartilhar os problemas enfrentados no CLS e solicitar a realização de uma eleição, 
o que foi atendido. Houve divulgação do processo eleitoral e as cartilhas distribuídas cumpriram papel informativo. Nas rodas de conversa também foram esclarecidas as dúvidas sobre questões relacionadas à participação no processo eleitoral e aos poderes de um conselho. Os líderes religiosos reforçaram o convite à população nas demais reuniões.

Ademais, a eSF orientou a população durante as visitas e procedimentos e convidou todos a participarem. De modo geral, o nível de conhecimento da população sobre os serviços ofertados pelo SUS na APS e os mecanismos de participação social ainda são limitados, devido a fatores como a obtenção de informações de forma confusa e contraditória que impossibilita a realização de escolhas e empoderamento dos usuários ${ }^{13,15}$. Parte desse desconhecimento é agravado pelo predomínio da ideia hospitalocêntrica, focada na doença, em desencontro com os princípios da APS, de saúde centrada na pessoa ${ }^{16}$. Assim, percebe-se a necessidade de melhorias no compartilhamento de informações com a população, para que seja participante ativa nos mecanismos de transformação do sistema de saúde e da realidade atual, baseado nas diretrizes do SUS ${ }^{17}$.

Ao longo da vivência da inserção no cotidiano dos serviços de saúde, permitida por meio da experiência do PET-Saúde/GraduaSUS, foi possível compreender e sentir desafios enfrentados na implantação do projeto, como o tempo reduzido para execução das ações, a limitação de apoio financeiro para o desenvolvimento e distribuição dos materiais, a dificuldade de tornar a ação contínua e de torná-la ampliada a outros territórios e a ausência de equipamentos como escolas ou praças para proporcionar o alcance de mais usuários. Além disso, ao decorrer da realização das rodas de conversa, toda a equipe precisou ser mobilizada para as ações de prevenção e/ou controle da febre amarela devido à epidemia, mudando a prioridade de atuação do grupo tutorial. Esses desafios refletem a realidade do contexto do SUS em sua complexidade e exigiram pensar novos caminhos e novas possibilidades, o que culminou com aprendizado e desenvolvimento de habilidades.

Por outro lado, alguns fatores favoreceram a resolução de problemas e a realização das ações, como o acesso facilitado às lideranças locais, especialmente religiosas; a existência de locais amplos com infraestrutura para a realização das oficinas, como as igrejas; e o envolvimento e empenho da eSF em todo o processo, inclusive na divulgação das oficinas e demais informações à população.

\section{O movimento PET-Saúde/GraduaSUS}

O PET-Saúde/GraduaSUS permitiu que diversas concepções fossem elencadas para a troca de saberes multiprofissionais e culturais, o que possibilitou avanços na compreensão das necessidades de saúde da população local e do próprio serviço de 
saúde. A identificação dos problemas, enumeração das prioridades e realização de atividades efetivas que pudessem minimizar os nós críticos foram de fundamental importância, pois proporcionaram um processo reflexivo entre os profissionais da ESF e maior aproximação do serviço com a comunidade.

A população foi informada sobre seus direitos e deveres enquanto agentes atuantes responsáveis pela saúde e pôde discutir sobre esses temas nas rodas de conversa, para que as reflexões repercutissem em maior participação e mais transparência nas ações de saúde. As ações do programa também permitiram despertar, na equipe de saúde, a necessidade de ampliar o trabalho de conscientização da população, nas visitas domiciliares, consultas ou em eventos realizados na comunidade.

Além da importância para o serviço e comunidade, o PET-Saúde/GraduaSUS trouxe grandes benefícios à formação acadêmica de seus integrantes, pois permitiu a ampliação da visão referente ao sistema de saúde, a partir do aprendizado extramuros. O contato prático com diferentes saberes e profissionais permitiu agregar novos conhecimentos que não são adquiridos no currículo tradicional. As ferramentas utilizadas no processo capacitaram e desafiaram os futuros profissionais a construírem novas formas de olhar, refletir e intervir na realidade, contribuindo para o amadurecimento profissional e pessoal.

\section{CONCLUSÃO}

A experiência de integração entre a academia e o cotidiano de trabalho no SUS mostrou-se fundamental por apresentar resultados positivos de melhorias para a população por intermédio da identificação de nós críticos, do estreitamento de diálogo entre profissionais, estudantes e usuários na elaboração de estratégias de mudanças e da intervenção realizada. Os discentes tiveram uma experiência ímpar de vivência e conhecimento prático na APS, de interlocução com atores e possibilidade de explorar os equipamentos sociais da área de atuação da ESF.

Para que seja alcançado maior conhecimento da população sobre saúde e sobre o SUS, é necessário um trabalho permanente de informação e envolvimento com a comunidade, incentivando sua participação ativa na rotina da UAPS. Além disso, torna-se relevante a elaboração e execução de estratégias de educação permanente, com objetivo de aprimorar a comunicação com a comunidade local e empoderar os usuários.

Os resultados desta experiência podem ser expandidos com o estreitamento da relação entre ensino, serviço e comunidade, (re)criando um fluxo contínuo de conhecimento e experimentações, culminando no maior atendimento às necessidades de saúde locais e fortalecimento do SUS. 


\section{FINANCIAMENTO}

A presente experiência contou com apoio financeiro do Ministério da Saúde, por meio de bolsas para tutores, preceptores e discentes do PET-Saúde/GraduaSUS, segundo aprovação pela portaria no 165, de 04 de abril de 2016.

\section{REFERÊNCIAS}

1. Ceccim RB. Educação Permanente em Saúde: descentralização e disseminação de capacidade pedagógica na saúde. Ciênc. saúde coletiva. 2005 dez; 10(4): 975-86.

2. Ministério da Saúde (BR), Ministério da Educação. Portaria Interministerial no 1.802, de 26 de agosto de 2008. Institui o Programa de Educação pelo Trabalho para a Saúde - PET - Saúde. Brasília: Ministério da Saúde; 2008.

3. Secretaria de Gestão do Trabalho e da Educação na Saúde (BR). Edital $n^{\circ} 13$, de 28 de setembro de 2015 seleção para o programa de educação pelo trabalho para a saúde PET-Saúde/GraduaSUS - 2016/2017. Brasília: Brasil; 2015.

4. Agostinho M, Oliveira M, Pinto M, Balardin G, Harzheim E. Autopercepção da Saúde entre usuários da Atenção Primária em Porto Alegre, RS. Revista Brasileira de Medicina da Família e Comunidade. 2010 mar; 5(17):9-15.

5. Ministério da Saúde. Política Nacional de Atenção Básica. Portaria n².488 de 21 de outubro de 2011. Brasília. 2011.

6. Matus C. Política, planejamento e governo. Brasília: IPEA; 1993.

7. César FIG. Ferramentas Básicas da Qualidade: instrumentos para gerenciamento de processos e melhoria continua. 1a edição. São Paulo: Biblioteca24horas; 2011.

8. Ferreira MEV, Schimith MD, Cáceres NC. Necessidades de capacitação e aperfeiçoamento dos profissionais de equipes de saúde da família da 4a Coordenadoria Regional de Saúde do Estado do Rio Grande do Sul. Ciênc. saúde coletiva. 2010 ago; 15(5):2611-20.

9. Besen CB, Netto MS, Da Ros MA, Silva FW, Silva CG, Pires MF. A estratégia saúde da família como objeto de educação em saúde. Saude soc. 2007 abr; 16(1):57-68.

10. Martins J, Garcia J, Passos A. Estratégia saúde da família: população participativa, saúde ativa. Enfermagem Integrada. 2008 maio; 1(1).

11. Barbosa P, Alonso R, Viana F. Aprendendo Ecologia Através de Cartilhas. 2oㅡ Congresso Brasileiro de Extensão Universitária. Belo Horizonte: Universidade Federal de Minas Gerais; 2004. 
12. Torres GV, Enders BC. Atividades educativas na prevenção da AIDS em uma rede básica municipal de saúde: participação do enfermeiro. Rev. Latino-Am. Enfermagem. 1999 abr; 7(2):71-7.

13. Vázquez ML, Silva MRF, Gonzalez ESC, Diniz AS, Pereira APC, Veras ICL, et al . Nível de informação da população e utilização dos mecanismos institucionais de participação social em saúde em dois municípios do Nordeste do Brasil. Ciênc. saúde coletiva [Internet]. 2005 maio; 10(Suppl):141-55.

14. Silva AX, Cruz EA, Melo V. A importância estratégica da informação em saúde para o exercício do controle social. Ciênc. saúde coletiva. 2007 jun; 12(3):683-8.

15. Viegas APB, Carmo RF, Luz ZMP. Fatores que influenciam o acesso aos serviços de saúde na visão de profissionais e usuários de uma unidade básica de referência. Saúde soc. 2015 mar; 24(1):100-12.

16. Carvalho STRF, Caccia-Bava MCGG. Conhecimentos dos usuários da Estratégia Saúde da Família sobre a fisioterapia. Fisioter. mov. 2011 dez; 24(4):655-64.

17. Martins PC. Controle social no Sistema Único de Saúde: análise da participação social e empoderamento da população usuária do sistema sanitário. Hist. cienc. Saúde -Manguinhos. 2008 mar; 15(1):238-238.

Submissão: abril de 2019.

Aprovação: julho de 2020. 